\title{
A Retrospective Study of Palliative Cisplatin-Based Doublet Chemotherapy for Malignant Pleural Mesothelioma
}

\author{
Steffen Filskov Sorensen ${ }^{1}$, Azza Ahmed Khalil ${ }^{1} \&$ Peter Meldgaard ${ }^{1}$ \\ ${ }^{1}$ Department of Oncology, Aarhus University Hospital, Noerrebrogade, Denmark \\ Correspondence: Steffen Filskov Sorensen, M.D., Department of Oncology, Aarhus University Hospital, \\ Noerrebrogade 44, 8000 Aarhus C, Denmark. Tel: 45-2993-7980. E-mail: stefso@rm.dk
}

Received: December 6, 2013 Accepted: January 2, 2014 Online Published: April 1, 2014

doi:10.5539/cco.v3n1p16

URL: http://dx.doi.org/10.5539/cco.v3n1p16

\begin{abstract}
Objectives: Cisplatin-based doublet-chemotherapy is the standard palliative treatment for malignant pleural mesothelioma (MPM) supplemented with palliative radiotherapy when indicated by symptoms. This work aims to study the epidemiologic characteristics and overall survival rates of patients with MPM treated with palliative chemotherapy in one institution year 2000 - 2010.

Materials and Methods: Review of journals. Data were structured using the Aarhus Lung Cancer Register and statistics were analyzed using SPSS software.

Results: The median age of the 80 patients ( 70 males and 10 females) at diagnosis was 63 years (range 40-80). $46 \%$ of the patients had epithelioid histological subtype. As first line treatment 21 patients received cisplatin/vinorelbine iv., 29 patients received cisplatin/pemetrexed and 11 patients received pemetrexed as monotherapy. Median overall survival (mOS) for the whole group was 13.1 months (95\% CI 10.1-16.2). We found no significant difference in mOS between patients treated with cisplatin/vinorelbine (mOS 17.0 months, 95\% CI 12.6-21.4) and cisplatin/pemetrexed (mOS 14.0 months, 95\% CI 8.9-19.1), $\mathrm{p}=0.598$. Patients with epithelioid subtype had a significantly better mOS (15.2 months, 95\% CI 11.6-18.8) compared to patients with non-epithelioid subtype ( 8.9 months, $95 \%$ CI 5.6-12.2), $\mathrm{p}=0.026$.

Conclusion: Subtype of histology is significantly associated with survival in MPM. Patients with epithelioid histology have a better prognosis than patients with non-epithelioid subtype. Our results show no significant difference in overall survival in patients who received different cisplatin-based doublet-regimes. The survival rates in this retrospective study are comparable to other published data. Randomized trials exploring cisplatin-based doublet regimens like cisplatin/pemetrexed and cisplatin/vinorelbine po. are needed.
\end{abstract}

Keywords: malignant pleural mesothelioma, palliative chemotherapy, palliative radiotherapy, cisplatin, pemetrexed, vinorelbine

\section{Introduction}

Malignant Pleural Mesothelioma (MPM) is a rare but highly aggressive cancer with a poor prognosis. In Europe approx. 5000 new cases occur yearly, probably resulting in more than 250.000 deaths over the next 40 years (Peto, Decarli, La Vecchia, Levi, \& Negri, 1999). MPM is associated with exposure to asbestos (Carbone, Kratzke, \& Testa, 2002). The success of curatively intended trimodality therapy, which includes major surgery (extrapleural pneumonectomy, EPP) combined with neoadjuvant chemotherapy and adjuvant radiotherapy, is limited, and is therefore only recommended for a highly selected group of patients. Pleurectomy/decortication $(\mathrm{P} / \mathrm{D})$ is another surgical treatment option that seems to improve survival (Kaufman \& Flores, 2011; Stahel, Weder, Lievens, Felip, \& ESMO Guidelines Working Group, 2010). As a result of these circumstances the majority of patients with MPM are treated with palliative chemotherapy and radiotherapy. Cisplatin, pemetrexed and vinorelbine are some of the previously studied chemotherapeutics in MPM. Other active drugs include raltitrexed and gemcitabine (Ellis et al., 2006; Tsao, Wistuba, Roth, \& Kindler, 2009). Cisplatin acts by interaction with DNA and forms DNA adducts, primarily intrastrand crosslink adducts leading to apoptosis. Pemetrexed is a multitargeted antifolate that inhibits thymidylate synthase, glycinamide ribonucleotide formyltransferase and dihydrofolate reductase, which leads to inhibition of the synthesis of thymidine and purine nucleotides. Vinorelbine is a semisynthetic vinca alkaloide synthetized on the basis of Catharanthus Roseus. The 
drug acts primarily by interaction with microtubule dynamics, which leads to mitotic arrest or cell death. Among others, older age, poor performance status, male gender and non-epithelioid subtype are found to be poor prognostic factors (Edwards et al., 2000; Nojiri et al., 2011).

Due to the relatively small number of patients diagnosed with MPM, only few randomized trials concerning palliative chemotherapy exists. Combination chemotherapy have been reported having higher response rates than single agent therapy, and platinum-containing regimes had better outcomes compared to non-platinum containing regimes (Ellis et al., 2006; Porpodis et al., 2013). A landmark randomized phase III trial $(n=456)$ by Vogelzang et al. demonstrated that the combination of cisplatin and pemetrexed was superior to cisplatin alone when given to chemotherapy-naïve patients with MPM (mOS 12.1 months vs. 9.3 months, $p=0.020$, response rate $41.3 \%$ vs. 16.7\%) (Vogelzang et al., 2003). This platinum-based doublet-regime is now established as the standard-of-care frontline regime against which other frontline regimes are evaluated. The combination cisplatin/vinorelbine iv. has been studied in a phase II study, where a mOS of 16.8 months (range 0.5-46.4+) and a response rate of 29.8\% was determined (Sorensen, Frank, \& Palshof, 2008). As second line treatment, monotherapy with vinorelbine is an option based on a study finding the drug moderately active and with acceptable toxicities (Stebbing et al., 2009).

The aim of this retrospective study is to explore the tumor and patient characteristics and survival rates related to type of treatment in a cohort of patients with inoperable MPM referred to Aarhus University Hospital in Denmark year 2000-2010. Our hypothesis is that the median overall survival rates are comparable for the two cisplatin-based doublet-regimens explored in this study. We also expect older age, male gender and non-epithelioid histology to be significant factors of poor prognosis.

\section{Materials and Methods}

At the Department of Oncology at Aarhus University Hospital (Denmark) the standard first line palliative chemotherapy until 2007 was cisplatin $\left(100 \mathrm{mg} / \mathrm{m}^{2}\right.$ every 4 weeks $)$ and vinorelbine $\left(25 \mathrm{mg} / \mathrm{m}^{2}\right.$ iv. given weekly). The standard first line treatment after 2007 has been cisplatin $\left(75 \mathrm{mg} / \mathrm{m}^{2}\right.$ every 3 weeks) and pemetrexed $\left(500 \mathrm{mg} / \mathrm{m}^{2}\right.$ every 3 weeks supplemented with folic acid and vitamin B-12). Patients with contraindications for cisplatin were treated with pemetrexed $\left(500 \mathrm{mg} / \mathrm{m}^{2}\right.$ every 3 weeks) as monotherapy in first line. Monotherapy with vinorelbine $\left(25 \mathrm{mg} / \mathrm{m}^{2}\right.$ iv. given weekly or $60 / 80 \mathrm{mg} / \mathrm{m}^{2}$ po. day 1 and 8$)$ was used as second line treatment, alternatively pemetrexed $\left(500 \mathrm{mg} / \mathrm{m}^{2}\right.$ every 3 weeks) if the patient was unexposed to this drug. If severe thoracic pain or symptoms of airway compression was conspicuous, palliative radiotherapy (20 Gy/4 fractions, 15 Gy/3 fx or $8 \mathrm{~Gy} / 1 \mathrm{fx}$ ) was offered prior to or concomitant with the initiation of palliative chemotherapy.

A total of 80 consecutive patients diagnosed with inoperable malignant pleural mesothelioma, who referred to Department of Oncology at Aarhus University Hospital for palliative treatment in the period 2000 and 2010, were reviewed. Disease stage was classified according to the Staging Manual in Thoracic Oncology 2009 by IASLC. Response to treatment and subsequent determination of disease control rate (complete response + partial response + stable disease) and response rate (complete response + partial response) was based upon the author's review of the imaging reports using the modified RECIST criteria for response to treatment in MPM (Byrne \& Nowak, 2004). The survival period was determined from the date of diagnosis confirmed by the pathologist until the date of death or April 11, 2013. Survival time was estimated using the Kaplan-Meier method, and log-rank test was used to compare survival between groups. A univariate Cox proportional hazards regression analysis was performed for each factor.

Data were structured using the Aarhus Lung Cancer Register and were analyzed using IBM ${ }^{\circledR}$ SPSS $^{\circledR}$ Statistics software version 21.0 for Windows.

\section{Results}

Baseline characteristics for the 80 included patients are listed in Table 1 . The majority were males $(88 \%)$ and the median age at diagnosis was 63 (range 40-80). The most frequent histological subtype was epithelioid (46\%). Most patients had an advanced stage of disease (39\% in stage IV). The majority were in good performance status (PS). $48 \%$ of the patients didn't have any comorbidity at all, and $16 \%$ had 2 comorbidities or more. The most frequent type of comorbidity was cardiovascular disease. $7.5 \%$ had cancer previously. 
Table 1. Baseline patient and tumor characteristics for all patients

\begin{tabular}{|c|c|}
\hline \multicolumn{2}{|c|}{ Patients and tumor characteristics (all patients) $N=80$} \\
\hline Median age at diagnosis (range) & $63(40-80)$ \\
\hline Gender (male/female) & $80(70 / 10)$ \\
\hline \multicolumn{2}{|l|}{ Histology } \\
\hline Epithelioid & $37(46 \%)$ \\
\hline Sarcomatoid & $14(17 \%)$ \\
\hline Biphasic & $23(30 \%)$ \\
\hline Subtype not specified & $6(7 \%)$ \\
\hline \multicolumn{2}{|l|}{ Stage } \\
\hline I & $15(19 \%)$ \\
\hline II & $12(15 \%)$ \\
\hline III & $21(26 \%)$ \\
\hline IV & $31(39 \%)$ \\
\hline Unknown & 1 \\
\hline \multicolumn{2}{|c|}{ Performance status at first line treatment } \\
\hline 0 & $25(31 \%)$ \\
\hline 1 & $41(51 \%)$ \\
\hline 2 & $7(9 \%)$ \\
\hline 3 & $1(1 \%)$ \\
\hline Unknown & $6(8 \%)$ \\
\hline \multicolumn{2}{|c|}{ Performance status at second line treatment } \\
\hline 0 & $4(12 \%)$ \\
\hline 1 & $24(71 \%)$ \\
\hline 2 & $5(15 \%)$ \\
\hline 3 & $1(3 \%)$ \\
\hline Unknown & 0 \\
\hline \multicolumn{2}{|l|}{ Comorbidities (number) } \\
\hline 0 & $38(48 \%)$ \\
\hline 1 & $29(36 \%)$ \\
\hline$\geq 2$ & $13(16 \%)$ \\
\hline \multicolumn{2}{|l|}{ Comobidity (type) } \\
\hline COPD & $4(5 \%)$ \\
\hline Cardiovascular & $22(27.5 \%)$ \\
\hline Diabetes Mellitus & $3(4 \%)$ \\
\hline Previous Cancer & $6(7.5 \%)$ \\
\hline Cerebrovascular & $4(5 \%)$ \\
\hline Others & $18(22.5 \%)$ \\
\hline
\end{tabular}

COPD: Chronic Obstructive Pulmonary Disease.

Cisplatin and vinorelbine was given to 21 patients and cisplatin and pemetrexed was given to 29 patients as the first palliative treatment. 14 patients received pemetrexed as monotherapy in first line (Table 2). None of the patients underwent surgery for MPM. Of the 11 patients who received palliative radiotherapy as first treatment, only 2 patients were treated with doublet chemotherapy afterwards. $20 \mathrm{~Gy}$ in 4 fractions was given to 5 patients as first treatment. The remaining 6 patients who received palliative radiotherapy as first treatment received other palliative radiation doses. The most common clinical indication for giving palliative radiotherapy was thoracic 
pain ( 5 out of 11 patients). 34 patients received second line treatment, predominately palliative radiotherapy (Table 2). Also for this group of patients, $20 \mathrm{~Gy}$ in 4 fractions was the most frequently used fractionation and thoracic pain was the most common symptom.

Table 2. Type of palliative treatment in first and second line

\begin{tabular}{lll}
\hline Palliative treatment & First treatment & Second treatment \\
\hline Cisplatin/vinorelbine & 21 & $1\left(1^{+}\right)$ \\
Cisplatin/pemetrexed & 29 & $1\left(1^{+}\right)$ \\
Pemetrexed & 14 & $10\left(1^{+}\right)$ \\
Vinorelbine & 0 & 2 \\
Palliative radiotherapy & 11 & 20 \\
Patients treated & 75 & 34 \\
No treatment & 5 & 46 \\
\hline
\end{tabular}

${ }^{+}$: Given after palliative radiotherapy in first line

The patient and tumor characteristics, categorized by type of treatment, are listed in Table 3 . The group who received pemetrexed as monotherapy were generally elderly (median age 75) and had a lower WHO performance status than the patients who received combination chemotherapy. The distribution of histological subtypes in the groups treated with cisplatin/pemetrexed or cisplatin/vinorelbine was significantly uneven, as more patients with a non-epithelioid histology were present in the cisplantin/pemetrexed group $(69 \%$ vs. $33 \%$, $\mathrm{p}=0.021)$. There was also a higher frequency of patients with advanced disease in the cisplatin/pemetrexed group than in the cisplatin/vinorelbine group ( $72 \%$ vs. $48 \%)$ although not reaching statistically significance $(\mathrm{p}=0.087)$.

Table 3. Patient and tumor characteristics according to type of 1st line treatment

\begin{tabular}{|c|c|c|c|c|c|}
\hline & $\begin{array}{l}\text { Cisplatin/Pemetrexed } \\
(n=29)\end{array}$ & $\begin{array}{l}\text { Cisplatin/Vinorelbine } \\
(n=21)\end{array}$ & p-value & $\begin{array}{l}\text { Pemetrexed } \\
(n=14)\end{array}$ & $\begin{array}{l}\text { Radiotherapy } \\
(n=11)\end{array}$ \\
\hline Median age (range) & $63(41-78)$ & $62(64-73)$ & & $75(53-80)$ & $63(48-80)$ \\
\hline$\leq 70$ & $23(79 \%)$ & $19(90 \%)$ & 0.168 & & 7 \\
\hline$>70$ & $6(21 \%)$ & $2(10 \%)$ & 0.441 & & 4 \\
\hline Male & $25(86 \%)$ & $19(90 \%)$ & 1.0 & 12 & 10 \\
\hline Female & $4(14 \%)$ & $2(10 \%)$ & & 2 & 1 \\
\hline PS 0 & $16(55 \%)$ & $7(33 \%)$ & 0.158 & $1(7 \%)$ & $1(9 \%)$ \\
\hline $\mathrm{PS} \geq 1$ & $13(45 \%)$ & $14(67 \%)$ & & $10(71 \%)$ & $5(45 \%)$ \\
\hline $\mathrm{PS} \geq 2$ & & & & $3(21 \%)$ & $4(36 \%)$ \\
\hline Unknown & & & & & $1(9 \%)$ \\
\hline Epithelioid & $9(31 \%)$ & $14(67 \%)$ & 0.021 & $6(43 \%)$ & $5(45 \%)$ \\
\hline Non-epithelioid & $20(69 \%)$ & $7(33 \%)$ & & $8(57 \%)$ & $6(55 \%)$ \\
\hline Stage & & & 0.087 & & \\
\hline I or II & $8(28 \%)$ & $11(52 \%)$ & & $4(29 \%)$ & $3(27 \%)$ \\
\hline III or IV & $21(72 \%)$ & $10(48 \%)$ & & $10(71 \%)$ & $8(73 \%)$ \\
\hline Comorbidity (number) & & & 0.093 & & \\
\hline 0 & $12(41 \%)$ & $14(67 \%)$ & & $3(21 \%)$ & $8(73 \%)$ \\
\hline$\geq 1$ & $17(59 \%)$ & $7(33 \%)$ & & $11(79 \%)$ & $3(27 \%)$ \\
\hline Full dose & $18(62 \%)$ & $7(33 \%)$ & 0.085 & & \\
\hline Reduced dose & $11(40 \%)$ & $14(67 \%)$ & & & \\
\hline
\end{tabular}

P-values were calculated using two-sided Fisher's exact test (Wilcoxon's signed-rank test was used for age). PS: Performance status. NOS: Non-otherwise specified. 
The median overall survival (mOS) for the whole cohort was 13.1 months $(95 \%$ confidence interval (CI) 10.1-16.2). The mOS estimates with regard to the type of first line chemotherapy reveals no significantly difference between the two cisplatin-based doublet regimens. The group treated with cisplatin/pemetrexed: had a mOS of 14.0 months (CI 8.9-19.1) compared to 17.0 months (CI 12.6-21.4) for patients in the group treated with cisplatin/vinorelbine, $\mathrm{p}=0.598$ (Figure 1). No statistically significant difference in mOS either occurred if the survival analysis were based on the time from of referral to the Department of Oncology or "date of first treatment" to death (data not shown). Disease control rate (DCR) (complete response + partial response + stable disease) was $59 \%$ for patients treated with cisplatin/pemetrexed and $48 \%$ for patients treated with cisplatin/vinorelbine. However, the difference was not statistically significant, $\mathrm{p}=0.388$. Response rates (complete response + partial response) were $28.6 \%$ and $17 \%$ respectively, also not reaching statistically significance, $\mathrm{p}=0.712$. The 14 patients who were treated with pemetrexed as monotherapy in first line had a mOS of 7.5 months (CI 4.1-10.8), and the 11 patients who received radiotherapy as the first treatment had a mOS of 13.5 months (CI 2.6-24.4). Only 3 of these patients were offered palliative chemotherapy afterwards (Table 2). The group of patients with epithelioid subtype had a mOS of 15.2 months (CI 11.6-18.8) in contrast to the group with non-epithelioid subtype (biphasic + sarcomatoid + subtype not specified) whose mOS was 8.9 months (CI 5.6-12.2). This result was statistically significant, $\mathrm{p}=0.026$ (Figure 2). Non-epithelioid subtype was associated with statistically significant HR of $1.779(\mathrm{p}=0.028)$ in the univariate analysis, but no significant hazard ratios were found for any of the other potential prognostic factors (Table 4).

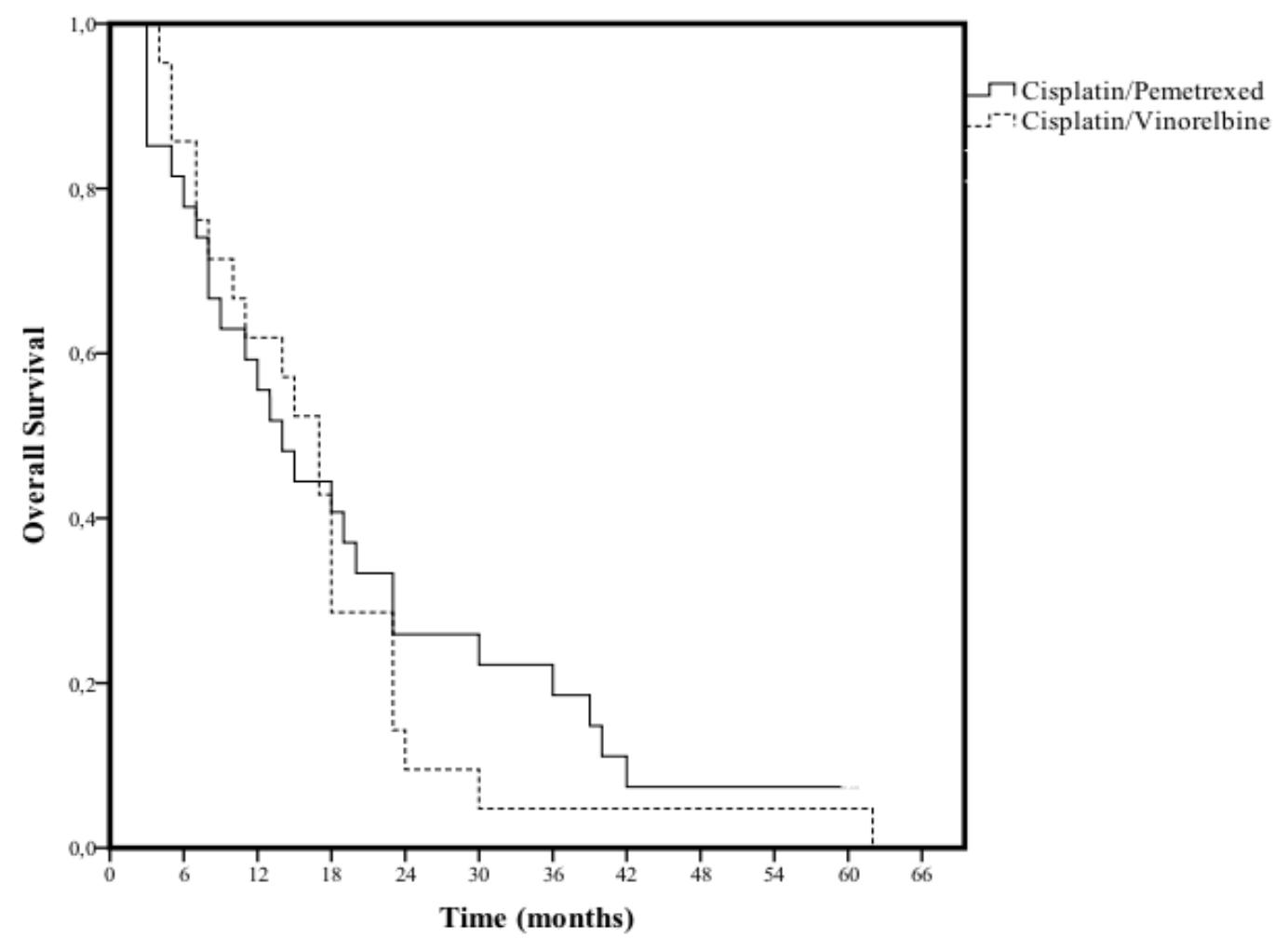

Figure 1. Kaplan-Meier survival curves of patients treated with cisplatin/pemetrexed $(n=21)$ or cisplatin/vinorelbine $(n=29)$ as first line palliative chemotherapy $(p=0.598)$ 


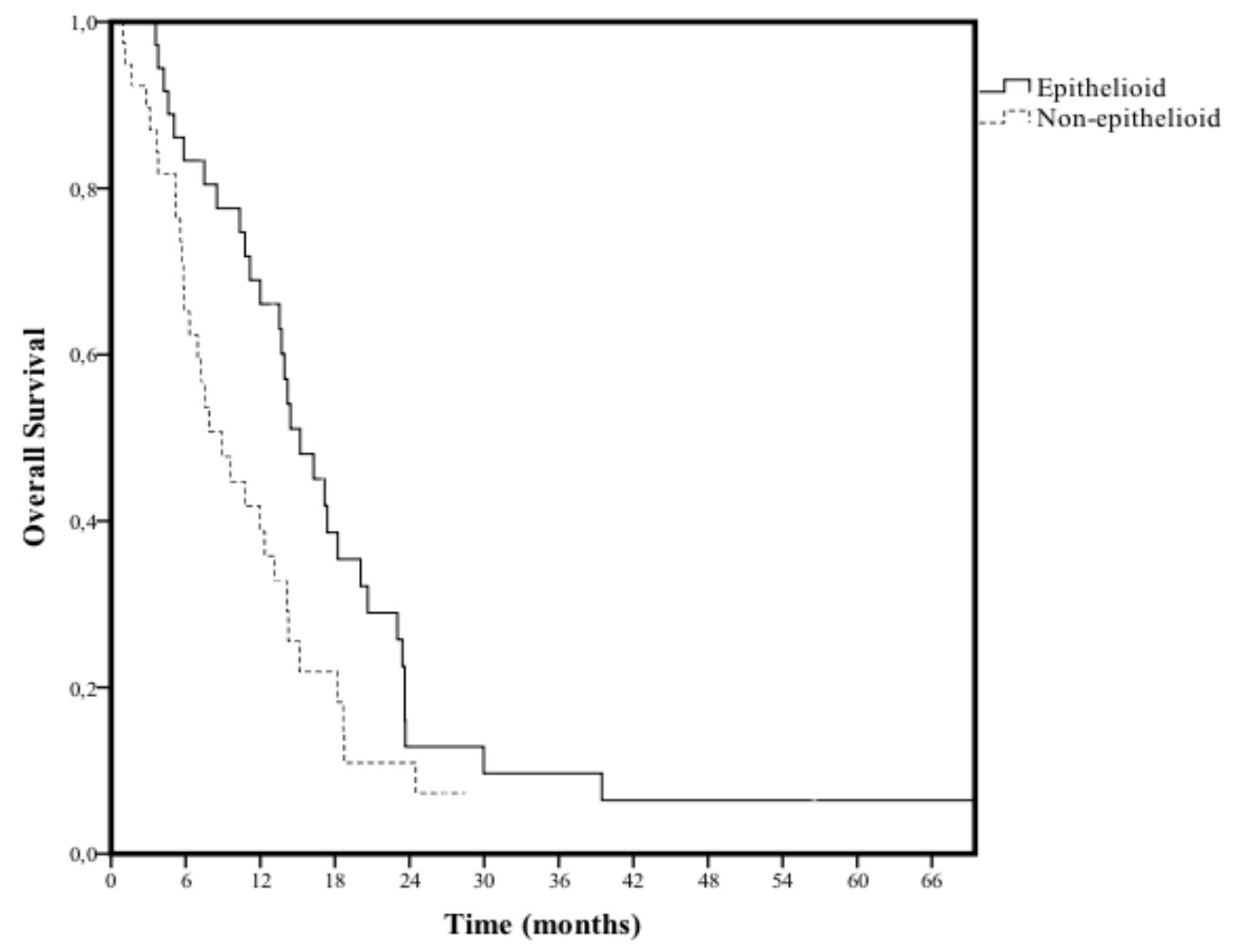

Figure 2. Kaplan-Meier survival curves of patients with epithelioid subtype ( $\mathrm{n}=37)$ and non-epithelioid subtype $(\mathrm{n}=43)(\mathrm{p}=0.026)$

Table 4. Cox regression univariate analysis

\begin{tabular}{lll}
\hline Variable & HR $(\mathbf{9 5 \%}$ CI) & p-value \\
\hline 1 or more comorbid diseases & $1.27(0.768-2.092)$ & 0.353 \\
Non-epithelioid subtype & $1.779(1.064-2.976)$ & 0.028 \\
Male gender & $1.097(0.501-2.402)$ & 0.818 \\
Stage III or IV & $1.019(0.626-1.659)$ & 0.939 \\
Age $>70$ & $1.30(0.705-2.405)$ & 0.395 \\
Palliative radiotherapy as first treatment & $0.928(0.470-1.835)$ & 0.831 \\
\hline
\end{tabular}

HR: Hazard ratio. CI: Confidence interval.

\section{Discussion}

In our single-institution retrospective study no statistically significant difference in overall survival, disease control rate or response rate between the platinum-based doublet regimens cisplatin/pemetrexed and cisplatin/vinorelbine iv., as first line palliative chemotherapy for MPM, was determined. Non-epithelioid histology predicted a statistically significant worse prognosis compared to epithelioid subtype. These results confirmed the initial hypothesis of our study, but older age and gender were not found to be significant prognostic factors.

The current recommendation of cisplatin/pemetrexed as first line palliative chemotherapy for MPM is mainly based on one randomised phase III trial, where this doublet regimen is compared to cisplatin monotherapy(Ellis et al., 2006; Obasaju et al., 2007; Santoro et al., 2008; Stahel et al., 2010; Vogelzang et al., 2003). To our knowledge, no randomized studies exists evaluating cisplatin-based doublet regimens against each other. A recently published retrospective study comparing pemetrexed- versus non-pemetrexed-containing doublet chemotherapy for the treatment of MPM in 48 patients, found median OS rates of 17.8 months and 17.0 months respectively $(\mathrm{p}=0.65)$. The patients and tumor characteristics were well balanced between the two groups also in 
terms of histological subtype. However, patients undergoing surgery (EPP or P/D) were also included in this study, potentially giving bias to the survival estimates (Higashiguchi et al., 2012). Lee et al. retrospectively compared patients with inoperable MPM either treated with cisplatin/pemetrexed $(n=40)$ or cisplatin/ gemcitabine $(\mathrm{n}=41)$, and determined median overall survival rates of 11.2 and 10.7 months respectively (no confidence intervals reported) (Lee, Murray, Anderson, Rao, \& Bishop, 2009). None of the mentioned retrospective studies evaluated response rates to chemotherapy. A phase II study, exploring the activity of cisplatin/vinorelbine iv. as first-line palliative chemotherapy for inoperable MPM in 54 patients, reported a mOS of 16.8 months (range $0.5-46.4+$ months) and a response rate of $29.6 \%$ (Sorensen et al., 2008). The study by Vogelzang et al. determined a mOS rate of 12.1 months (CI 10.0-14.4) for the cisplatin/pemetrexed group, and a response rate of $41.3 \%$ (Vogelzang et al., 2003). Toxicity was not evaluated in the present study, but the data from the studies exploring cisplatin/pemetrexed and cisplatin/vinorelbine does not indicate any major differences in the overall toxicity. The previously published mOS estimates and response rates, and the results from our analysis cannot be directly compared due to differences in study design, but they seem reasonably comparable.

The poor prognosis of the group treated with pemetrexed monotherapy in first line could be explained from the clinical characteristics of these patients. They were elderly, had more comorbidity and a higher PS than the patients who received doublet chemotherapy. The physician's choice of treatment was of course based upon these factors. Initial need for palliative radiotherapy due to severe symptoms, doesn't seem to indicate a bad prognosis, since the median overall survival rate for this group was comparable to the survival estimates for patients who received doublet chemotherapy. Patients who received radiotherapy as first line treatment had a non-significant HR of 0.928 (Table 4). However, any conclusion regarding this aspect cannot be drawn, as symptoms weren't explored in our study and due to the small sample size.

The limitations of our work are related to its retrospective design and the small number of patients. The cohort is unselected and therefore differences between groups, potentially affecting the survival statistics comparing types of treatment, occurs. An example of this is the frequency of epithelioid and non-epithelioid subtypes in the cisplatin/pemetrexed and the cisplatin/vinorelbine groups $(\mathrm{p}=0.021)$ (Table 3). The higher number of individuals in the cisplatin/vinorelbine group with non-epithelioid subtype could give these patients a worse outcome. Hence, the survival data of our study should be interpreted with caution, although a trend toward comparable survival rates for the two cisplatin-based doublet-regimens (cisplatin/pemetrexed vs. cisplatin/vinorelbine) can be drawn.

Targeted drugs have come into clinical use for many types of cancers like e.g. tyrosine kinase inhibitors (TKIs) for EGFR-mutated non-small cell lung cancer, but for MPM, conventional chemotherapy is still the treatment available for the vast majority of patients. Due to the potential biases of retrospective studies, randomized phase III studies evaluating the efficacy and feasibility of cisplatin-based doublet-regimens like cisplatin/pemetrexed and cispltin/vinorelbine in inoperable MPM are needed. After the introduction of a peroral formulation of vinorelbine for the treatment of e.g. NSCLC (Bartsch, 2006; Depierre et al., 2001; Gralla et al., 2007; Jassem et al., 2001), the cisplatin/vinorelbine po. combination might be a feasible and more administration-friendly alternative to cisplatin/pemetrexed in patients with inoperable MPM.

\section{Conclusion}

In conclusion we find that subtype of histology appears to be significantly associated with survival. Patients with epithelioid histology may have a better prognosis than patients with non-epithelioid subtype. Our results show no significant difference in overall survival in patients who received different cisplatin-based doublet-regimes, but due to an uneven distribution of histological subtypes between the groups, this result has to be interpreted with caution. MPM is still a disease with a poor prognosis, and the survival rates in this retrospective study are comparable to other published data.

The therapeutic ceiling for conventional chemotherapy in terms of survival improvement in patients with inoperable MPM seems to be reached, but further improvements in feasibility and ways of administration of the different doublet regimens could be obtained through the initiation of randomized trials.

\section{References}

Bartsch, V. (2006). Oral vinorelbine: pharmacology and treatment outcome in non-small cell bronchial carcinoma and breast carcinoma. [Orales Vinorelbin: Pharmakologie und Behandlungsergebnisse beim nichtkleinzelligen Bronchialkarzinom und Mammakarzinom] Onkologie, 29(Suppl 1), 1-28. http://dx.doi.org/10.1159/000091889

Byrne, M. J., \& Nowak, A. K. (2004). Modified RECIST criteria for assessment of response in malignant pleural mesothelioma. Annals of Oncology : Official Journal of the European Society for Medical Oncology ESMO, 
15(2), 257-260.

Carbone, M., Kratzke, R. A., \& Testa, J. R. (2002). The pathogenesis of mesothelioma. Seminars in Oncology, 29(1), 2-17.

Depierre, A., Freyer, G., Jassem, J., Orfeuvre, H., Ramlau, R., Lemarie, E., ... Trillet-Lenoir, V. (2001). Oral vinorelbine: feasibility and safety profile. Annals of Oncology : Official Journal of the European Society for Medical Oncology / ESMO, 12(12), 1677-1681.

Edwards, J. G., Abrams, K. R., Leverment, J. N., Spyt, T. J., Waller, D. A., \& O'Byrne, K. J. (2000). Prognostic factors for malignant mesothelioma in 142 patients: validation of CALGB and EORTC prognostic scoring systems. Thorax, 55(9), 731-735.

Ellis, P., Davies, A. M., Evans, W. K., Haynes, A. E., Lloyd, N. S., \& Lung Cancer Disease Site Group of Cancer Care Ontario's Program in Evidence-based Care. (2006). The use of chemotherapy in patients with advanced malignant pleural mesothelioma: a systematic review and practice guideline. Journal of Thoracic Oncology: Official Publication of the International Association for the Study of Lung Cancer, 1(6), 591-601.

Gralla, R. J., Gatzemeier, U., Gebbia, V., Huber, R., O'Brien, M., \& Puozzo, C. (2007). Oral vinorelbine in the treatment of non-small cell lung cancer: rationale and implications for patient management. Drugs, 67(10), 1403-1410.

Higashiguchi, M., Suzuki, H., Hirashima, T., Kobayashi, M., Goya, S., Okamoto, N., ... Kawase, I. (2012). A retrospective study of chemotherapy with and without pemetrexed in malignant pleural mesothelioma. Anticancer Research, 32(2), 609-613.

Jassem, J., Ramlau, R., Karnicka-Mlodkowska, H., Krawczyk, K., Krzakowski, M., Zatloukal, P., ... Depierr, A. (2001). A multicenter randomized phase II study of oral vs. intravenous vinorelbine in advanced non-small-cell lung cancer patients. Annals of Oncology : Official Journal of the European Society for Medical Oncology / ESMO, 12(10), 1375-1381.

Kaufman, A. J., \& Flores, R. M. (2011). Surgical treatment of malignant pleural mesothelioma. Current Treatment Options in Oncology, 12(2), 201-216. http://dx.doi.org/10.1007/s11864-011-0154-4

Lee, C. W., Murray, N., Anderson, H., Rao, S. C., \& Bishop, W. (2009). Outcomes with first-line platinum-based combination chemotherapy for malignant pleural mesothelioma: a review of practice in British Columbia. Lung Cancer (Amsterdam, Netherlands), 64(3), 308-313. http://dx.doi.org/10.1016/j.lungcan.2008.09.008

Nojiri, S., Gemba, K., Aoe, K., Kato, K., Yamaguchi, T., Sato, T., ... Kishimoto, T. (2011). Survival and prognostic factors in malignant pleural mesothelioma: a retrospective study of 314 patients in the west part of Japan. Japanese Journal of Clinical Oncology, 41(1), 32-39. http://dx.doi.org/10.1093/jjco/hyq159

Obasaju, C. K., Ye, Z., Wozniak, A. J., Belani, C. P., Keohan, M. L., Ross, H. J., ... Pemetrexed Expanded Access Program Investigators. (2007). Single-arm, open label study of pemetrexed plus cisplatin in chemotherapy naive patients with malignant pleural mesothelioma: outcomes of an expanded access program. Lung Cancer (Amsterdam, Netherlands), 55(2), 187-194. http://dx.doi.org/10.1016/j.lungcan.2006.09.023

Peto, J., Decarli, A., La Vecchia, C., Levi, F., \& Negri, E. (1999). The European mesothelioma epidemic. British Journal of Cancer, 79(3-4), 666-672. http://dx.doi.org/10.1038/sj.bjc.6690105

Porpodis, K., Zarogoulidis, P., Boutsikou, E., Papaioannou, A., Machairiotis, N., Tsakiridis, K., ... Zarogoulidis, K. (2013). Malignant pleural mesothelioma: current and future perspectives. Journal of Thoracic Disease, 5(Suppl 4), S397-S406. http://dx.doi.org/10.3978/j.issn.2072-1439.2013.08.08

Santoro, A., O'Brien, M. E., Stahel, R. A., Nackaerts, K., Baas, P., Karthaus, M., ... Manegold, C. (2008). Pemetrexed plus cisplatin or pemetrexed plus carboplatin for chemonaive patients with malignant pleural mesothelioma: results of the International Expanded Access Program. Journal of Thoracic Oncology: Official Publication of the International Association for the Study of Lung Cancer, 3(7), 756-763. http://dx.doi.org/10.1097/JTO.0b013e31817c73d6

Sorensen, J. B., Frank, H., \& Palshof, T. (2008). Cisplatin and vinorelbine first-line chemotherapy in non-resectable malignant pleural mesothelioma. British Journal of Cancer, 99(1), 44-50. http://dx.doi.org/10.1038/sj.bjc.6604421

Stahel, R. A., Weder, W., Lievens, Y., Felip, E., \& ESMO Guidelines Working Group. (2010). Malignant pleural mesothelioma: ESMO Clinical Practice Guidelines for diagnosis, treatment and follow-up. Annals of Oncology : Official Journal of the European Society for Medical Oncology/ESMO, 21(Suppl 5), v126-8. 
http://dx.doi.org/10.1093/annonc/mdq173

Stebbing, J., Powles, T., McPherson, K., Shamash, J., Wells, P., Sheaff, M. T., ... Steele, J. P. (2009). The efficacy and safety of weekly vinorelbine in relapsed malignant pleural mesothelioma. Lung Cancer (Amsterdam, Netherlands), 63(1), 94-97. http://dx.doi.org/10.1016/j.lungcan.2008.04.001

Tsao, A. S., Wistuba, I., Roth, J. A., \& Kindler, H. L. (2009). Malignant pleural mesothelioma. Journal of Clinical Oncology: Official Journal of the American Society of Clinical Oncology, 27(12), 2081-2090. http://dx.doi.org/10.1200/JCO.2008.19.8523

Vogelzang, N. J., Rusthoven, J. J., Symanowski, J., Denham, C., Kaukel, E., Ruffie, P., ... Paoletti, P. (2003). Phase III study of pemetrexed in combination with cisplatin versus cisplatin alone in patients with malignant pleural mesothelioma. Journal of Clinical Oncology: Official Journal of the American Society of Clinical Oncology, 21(14), 2636-2644. http://dx.doi.org/10.1200/JCO.2003.11.136

\section{Copyrights}

Copyright for this article is retained by the author(s), with first publication rights granted to the journal.

This is an open-access article distributed under the terms and conditions of the Creative Commons Attribution license (http://creativecommons.org/licenses/by/3.0/). 